\title{
The ASTRO-F (IRIS) Mission
}

\author{
Hiroshi Shibai \\ Nagoya University, Furo-cho, Chikusa-ku, Nagoya 464-8602, Japan
}

\begin{abstract}
ASTRO-F (IRIS) is the first Japanese satellite dedicated to infrared astronomy. The primary purpose of this project is to investigate the birth and evolution of galaxies in the early universe through deep, wide-field surveys at wavelengths ranging from 2 to 200 microns. The spatial resolution and the point source sensitivity are nearly the same as those of the aperture diffraction limit and the natural background and/or confusion limit, respectively. In the far-infrared wavelength band, $A S T R O-F$ will conduct an all-sky survey like the IRAS survey with several tens of times higher sensitivity and several times better spatial resolution. In the near- and mid-infrared, wide area sky-surveys will be conducted over pre-selected portions of the sky. In addition to these photometric surveys, low-resolution spectroscopic capabilities are available for all wavelength bands. The ASTRO-F mission will produce a fundamental database for the next generation of advanced observatories, for example FIRST, and NGST, and will complement the SIRTF mission by virtue of its wide sky coverage. The launch by an $\mathrm{M}-\mathrm{V}$ rocket is scheduled for February or March of 2004.
\end{abstract}

\section{Introduction}

Satellite-borne infrared telescopes have made remarkable contributions to recent advances in astronomy and astrophysics. The catalogs and sky images produced from the IRAS survey (Neugebauer et al. 1984) are among the most useful fundamental databases in all astronomy. The diffuse infrared maps made by DIRBE on COBE and the IRTS (Murakami et al. 1996) have been employed for determining the astrophysics of the interstellar medium and other phenomena. FIRAS on $C O B E$ gave us the most important spectrum of the Cosmic Microwave Background (CMB) radiation so far. ISO (Kessler 1996) was the first and the only infrared satellite of the observatory type, and executed numerous astronomical investigations of wide varieties.

Following these successful infrared satellites, the ASTRO-F (IRIS: the Infrared Imaging Surveyor) project is being developed as the first Japanese satellite dedicated to infrared astronomy, as one of the ASTRO-series projects of ISAS (Murakami 1998; Shibai 2000). This series is managed by the Institute of Space and Astronautical Science (ISAS), and is supported by scientists of Nagoya University, the University of Tokyo, and other universities and institutions in Japan, as well as by collaborations with foreign institutions and scientists. 


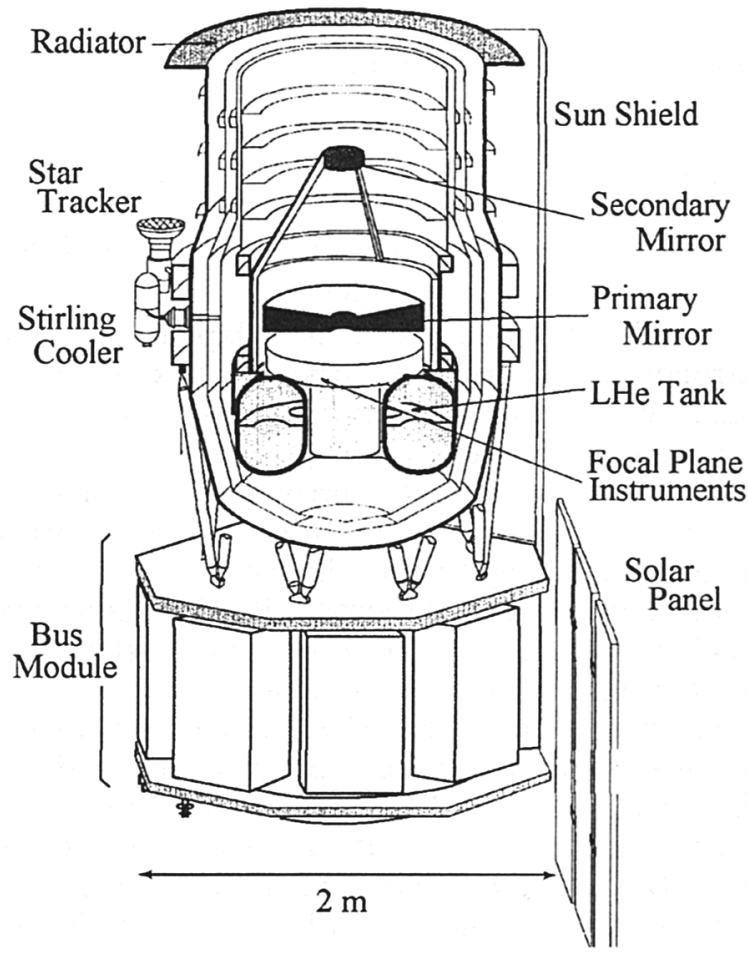

Figure 1. Cross-sectional view of the ASTRO-F (IRIS) spacecraft.
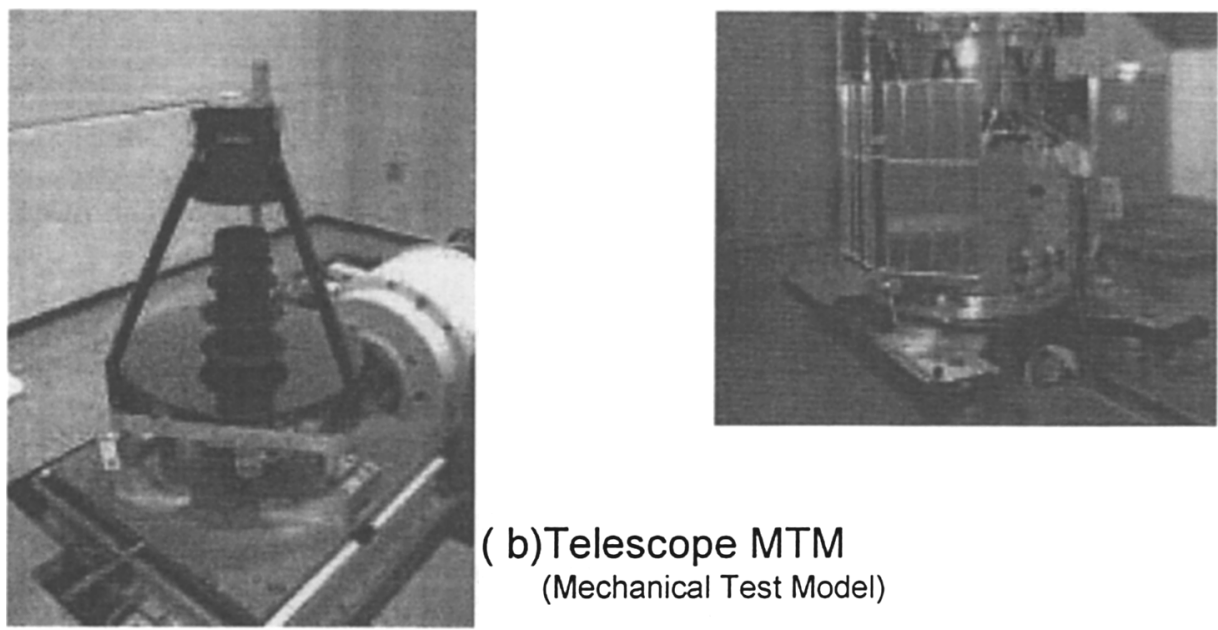

(b)Telescope MTM (Mechanical Test Model)

Figure 2. Mechanical test models of the ASTRO-F. (a) Cryostat, and (b) telescope. 
Table 1. Summary of the ASTRO-F (IRIS) Mission

\begin{tabular}{|c|c|}
\hline Cryogenics & $\begin{array}{l}170 \text { liter liquid helium cryostat }+ \text { Stirling } \\
\text { coolers }+ \text { radiative cooling }\end{array}$ \\
\hline Optical System & $\begin{array}{l}\text { Primary mirror aperture } 70 \mathrm{~cm} \text { at }<6 \mathrm{~K} \text {. } \\
\text { Ritchey-Chrétien } \mathrm{F} / 6 \text {. } \\
\text { Focal length } 4.2 \mathrm{~m} \text {. Image quality }<1 \text { arcsec } \\
\text { without diffraction effect }\end{array}$ \\
\hline \multicolumn{2}{|r|}{ (20) } \\
\hline $\begin{array}{l}\text { IRC } \\
\text { (Near/Mid IR Camera) }\end{array}$ & $\begin{array}{lc}\text { InSb }(512 \times 412) & 1.4 " / \text { pixel } \\
\text { Si:As-BIB }(256 \times 256) & 2.3 " / \text { pixel } \\
\text { Bands } 2.2,3.8,5,7,9,11,15,20,25 \mu \mathrm{m} \\
\text { Grism spectrometer }(\mathrm{R} \sim 50)\end{array}$ \\
\hline $\begin{array}{l}\text { FIS } \\
\text { (Far-Infrared Surveyor) }\end{array}$ & $\begin{array}{l}\text { Ge:Ga }(5 \times 20) \quad 30 " / \text { pixel } \\
\text { Stressed Ge:Ga }(5 \times 15) \quad 50 " / \text { pixel } \\
50-70,50-100,100-200,150-200 \mu \mathrm{m} \text { bands } \\
\text { Fourier spectrometer }(\mathrm{R} \sim 200)\end{array}$ \\
\hline Orbit & $\begin{array}{l}\text { sun-synchronous orbit at } 700-900 \mathrm{~km} \text { along } \\
\text { day/night border }\end{array}$ \\
\hline Attitude control & $\begin{array}{l}\text { pointing accuracy }<15 \text { arcsec } \\
\text { pointing stability }<1 \text { arcsec } / \text { minute }\end{array}$ \\
\hline $\begin{array}{l}\text { Observation Modes } \\
\text { Continuous survey }\end{array}$ & $\begin{array}{l}\text { constant scanning along a great circle perpen- } \\
\text { dicular to the Sun } \\
\text { full sky coverage in a half year }\end{array}$ \\
\hline Pointing & $\begin{array}{l}\text { deep survey with a fixed attitude }(\sim 10 \\
\text { minutes) } \\
\text { for IRC imaging and FIS spectroscopy }\end{array}$ \\
\hline Mission life & $\begin{array}{l}>500 \text { days (nominal cryogen life) } \\
\text { near-infrared observations can be continued } \\
\text { after the LHe run-out }\end{array}$ \\
\hline Total Mass & $960 \mathrm{~kg}$ (wet) \\
\hline
\end{tabular}




\section{Spacecraft and Cryostat}

Figure 1 shows a cross-sectional view of the ASTRO-F satellite. The satellite consists of the two parts, the mission part and the bus module. The bus module contains the service modules necessary for the operations and the survival of the spacecraft in orbit. The mission part consists of the cryostat containing the telescope, focal plane instruments, coolers, and so on. Figure 2 shows the ASTRO-F MTM (Mechanical Test Model).

The telescope is cooled down to $6 \mathrm{~K}$ to reduce instrumental thermal emission. The primary mirror has a $70 \mathrm{~cm}$ aperture, and is a newly developed, lightweight, $\mathrm{SiC}$ mirror (Onaka et al. 1998). The optical system is of the RitcheyChrétien type, and has a focal length of $4200 \mathrm{~mm}$. The goal is to achieve better than 1 arcsecond image quality at $6 \mathrm{~K}$ in orbit. A focus adjusting mechanism is installed at the secondary mirror assembly. The MTM of the telescope is shown in Figure 2.

$A S T R O-F$ will be the first example of a hybrid-type cryostat satellite incorporating a mechanical cooler as well as cryogen. Adopting this hybrid cooling system, we can install a large aperture telescope into a small cryostat. The telescope and the focal plane instruments are cooled down to a temperature of $2 \mathrm{~K}$ by super-fluid helium. The tank capacity is 170 liters. This value is considerably smaller than those of previous cooled satellites. By employing a mechanical cooling system (Stirling cycle cooler), most of the heat load from the cryostat's outer shell is absorbed and pumped away by the mechanical cooler system. The expected cryogen life is one and a half years. In addition to this, near-infrared observations can be continued using solely the mechanical cooling system even after the helium cryogen runs out.

An M-V rocket will launch $A S T R O-F$ into a sun-synchronous circular orbit at an altitude of $700-900 \mathrm{~km}$. The M-V solid fuel rocket is a new launch vehicle developed by ISAS. The last launch of the $\mathrm{M}-\mathrm{V}$ rocket unfortunately failed with the X-ray satellite, ASTRO-E on board. However, the cause of this failure has been carefully investigated and determined by ISAS. After a revision of the rocket design, the next M-V launch will take place in December 2002.

$A S T R O-F$ has two operating modes, a survey mode and a pointing mode. The survey mode is similar to that of IRAS. The ASTRO-F spacecraft rotates once per orbital revolution at a constant rate and traces a scanning path of great circles like IRAS. The difference is that IRAS was generally pointed in an anti-geocentric direction, while $A S T R O-F$ will always be pointed perpendicular to the radius vector from the Sun and nearest to the anti-geocentric direction on this great circle. As the result, the $A S T R O-F$ survey covers the whole sky in a half year with nodes at both zodiacal poles; the sky near these nodes is scanned many times. Table 1 summarizes the characteristics of the ASTRO-F (IRIS) mission.

Mechanical test models (MTM) of both the ASTRO-F spacecraft and telescope have been constructed and used for verification of the spacecraft design, as shown in Figure 2. The vibration level of solid-fueled rockets, such as the $\mathrm{M}-\mathrm{V}$ rocket, is generally larger than that of liquid-fueled rockets. Therefore, design and verification tests were carefully executed, and both models have already passed a mechanical environment test. We are now preparing a thermal environment test to be conducted toward the end of November, 2000. 


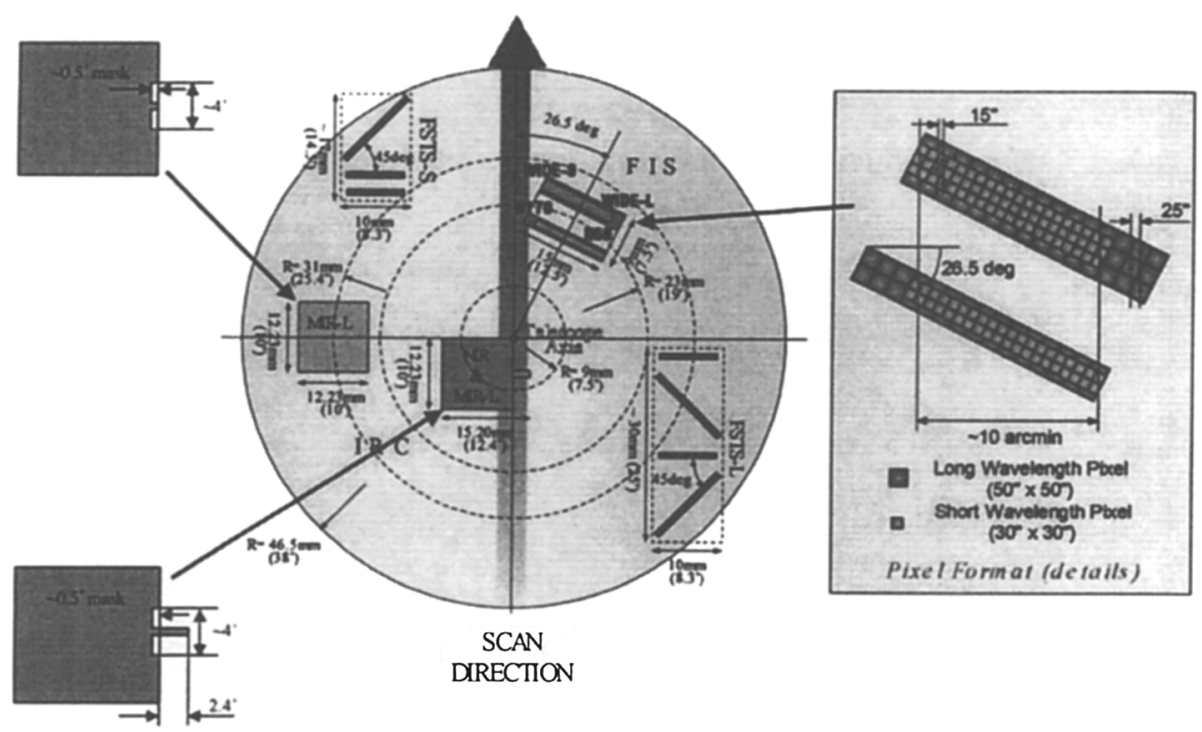

Figure 3. Configuration of each field of view in the telescope focal plane.

\section{Focal Plane Instruments}

Figure 3 shows the configuration of the focal plane. ASTRO-F has two scientific instruments, the Far-Infrared Surveyor (FIS) and the Infrared Camera (IRC). It also houses a focal plane star sensor (FSTS). They share the focal plane for optimal use of the light beam. The near-infrared and shorter mid-infrared channel of the IRC tap the region nearest the optical axis of the telescope to assure highest spatial resolution, while the longer mid-infrared channel, the FIS, and the FSTS make use of peripheral areas.

The IRC consists of three independent cameras covering the near- and midinfrared regions. The optics are designed with refractive elements of $\mathrm{Si}, \mathrm{Ge}$, KRS-5, and CsI; the design image quality is sharper than the size of individual pixels. The large format arrays of IRC (Matsuhara et al. 1998; Onaka et al. 1998) can cover wide fields with better spatial resolution than the present infrared cameras in space, and, by incorporating grisms, achieve highly efficient spectroscopic surveys. The low dispersion spectroscopic capability in the nearinfrared is unique and complements SIRTF. The arrays are state-of-the-art, and are optimized for low background condition in space. The optical configuration of the IRC is shown in Figure 4.

The FIS (Kawada 1998) is primarily designed for an IRAS-type all-sky survey in the wavelength region from 50 to $200 \mu \mathrm{m}$. Stressed and unstressed Ge:Ga large arrays were newly developed to cover this wavelength region. The former covers the region longer than $100 \mu \mathrm{m}$ (Figure 5), the latter the spectral range below $100 \mu \mathrm{m}$. The pixel sizes were determined to be nearly equal to the diffraction-limited resolution. Each array has one wide-band channel of three rows and one narrow-band channel of two rows. The pixel configurations 


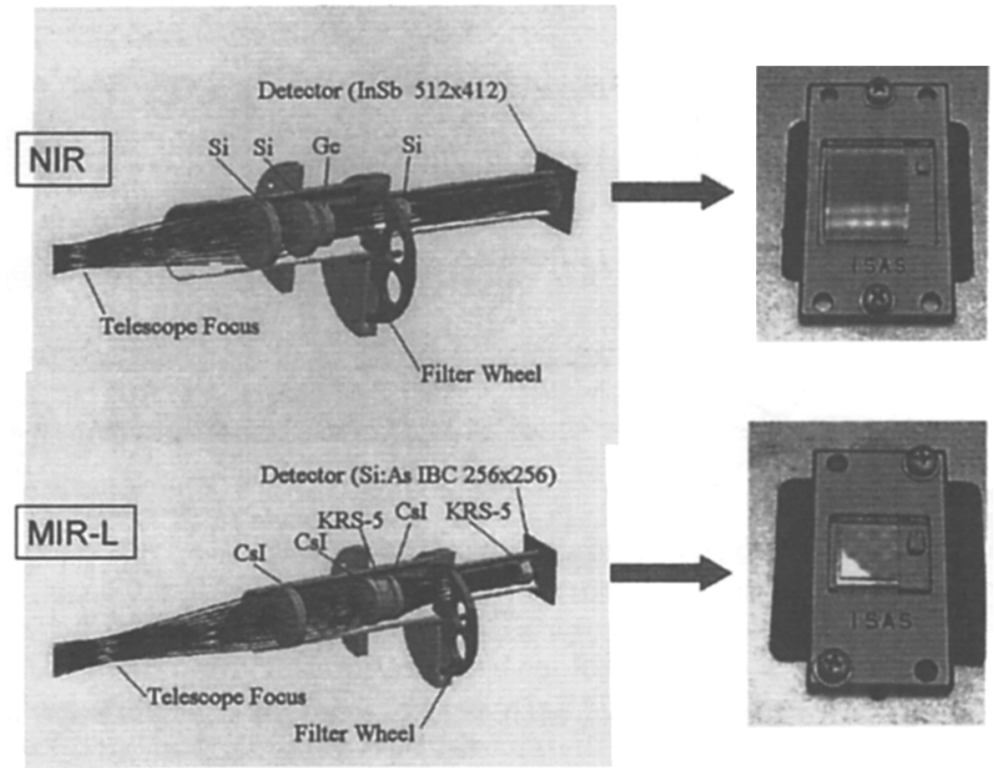

Figure 4. Optical systems and arrays of the infrared camera (IRC).
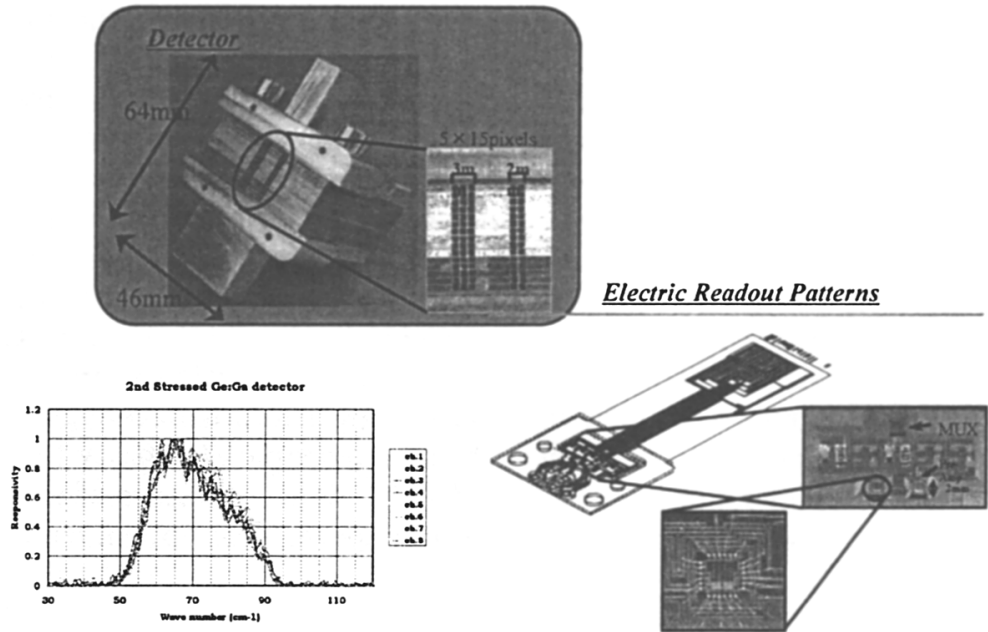

Figure 5. Stressed Ge:Ga array detector assembly (upper photograph). At lower left is the spectral response of the array; the lower right inset shows the structure of a single stage of the array. 


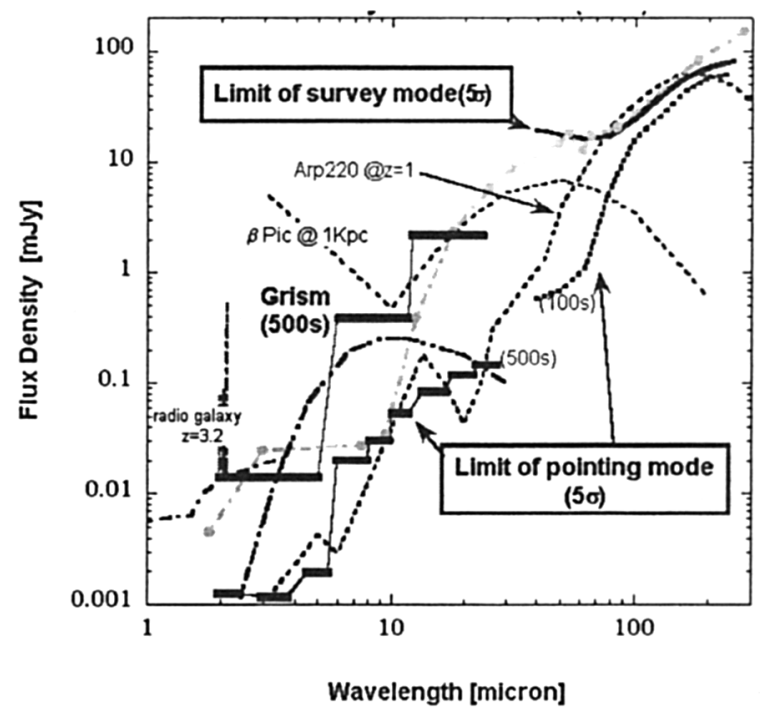

Figure 6. Point source sensitivity of the ASTRO-F (IRIS) mission

of both arrays are shown in Figure 3. The arrays are tilted by 26.5 degrees from the perpendicular to the scanning direction, which allows us to obtain sampling points by a spacing of a half of the pixel pitch for better resolution in the cross-scanning direction. In addition to the wide band all-sky survey, a Fourier transform spectroscope is incorporated for measurement of the SEDs of various objects and for detection of strong line emissions from distant starburst galaxies. The spectrometer is a Martin-Puplett interferometer that polarizes light; its spectral resolution is $0.2-0.3 \mathrm{~cm}^{-1}$.

The FSTS consists of two modules mounted directly on the focal plane. The role of this sensor is to produce time-sequence signals at I-band wavelengths in the survey mode. These provide the primary data for attitude reconstruction in the all-sky survey observing mode.

\section{Scientific Capability}

Figure 7 shows a comparison of fields-of-view and pixel configurations of the space infrared telescopes, IRAS, ISO, ASTRO-F (IRIS), and SIRTF. ASTRO$F$ has wider fields-of-view than $S I R T F$ and has better spatial resolution than $I R A S$ and ISO. This advantage is obtained by adopting state-of-the-art spaceoptimized arrays in the near- and mid-infrared and through use of newly developed far-infrared arrays, and is a most important feature for wide sky survey observations.

Two panels in Figure 8 show the observational capabilities of ASTRO-F. The upper shows the spectroscopic capabilities in each band. As shown, lowresolution spectroscopy is possible in all bands. SEDs (spectral energy distributions) of starburst galaxies as well as galactic star forming regions are efficiently obtained by this capability. The lower panel shows the presently planned sky 


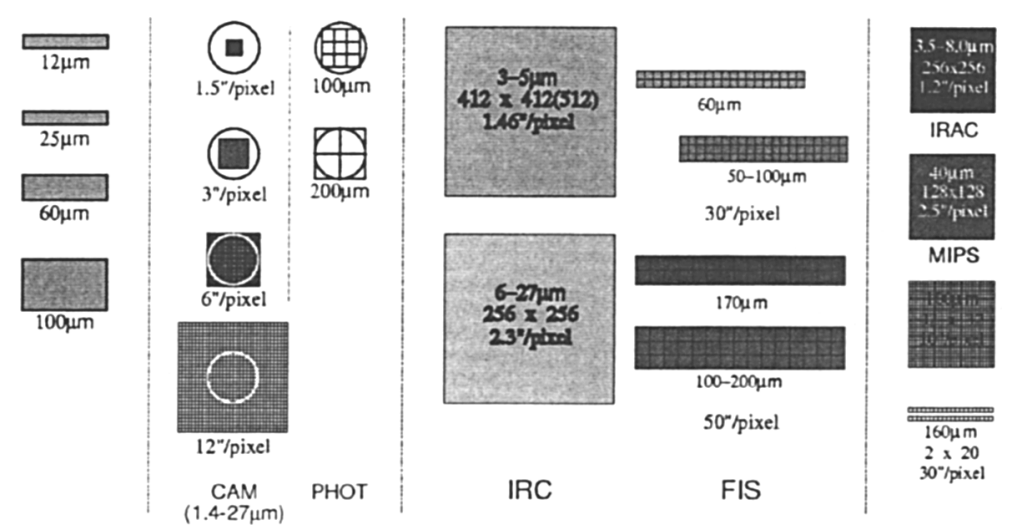

Figure 7. Comparison of array configurations of space-borne infrared telescopes

coverage. The whole sky is covered in the far-infrared wide-band survey. Up to 100 square degrees will be surveyed with IRC wide-band imaging, and on the order of 1 square degree can be covered with spectroscopy of both instruments. Most of these capabilities are unique.

\section{Operation}

$A S T R O-F$ is fundamentally a survey-type satellite on a polar orbit, as shown in Figure 9. Therefore, the cooled telescope must always be pointed away from the Earth. In the continuous survey mode for the all-sky survey, the telescope is scanning at a constant rate along the great circle perpendicular to the Sun. This will enable us to completely cover the sky during the first half year of the survey (Phase I). However, during passages of the South Atlantic Anomaly (SAA), the detectors will be useless due to impact of high-energy particles and to their after effects. In addition to this, the Moon will shine into the telescope when the Moon is within 33 degrees from the telescope axis. Due to these effects, and possibly due to other miscellaneous failures, malfunctions, calibrations, and maintenance operations, the whole sky cannot be covered within a half year. The parts of the sky which are not covered during this first half-year of the survey will be observed during the next half year and later (Phase II) while the cryogen lasts.

Pointing observations can be executed by staring at a certain direction for periods of ten minutes, as shown in Figure 9. The expected attitude stability is less than one arc second per one minute of time. The longest exposure of the IRC is 64 seconds, and so, the stability is sufficent even for pointing observations of the near-infrared channel. These pointing observations can be programmed mostly in Phase II. The maximum number of the pointing observation is three per orbit, and the total number of the ten-minute pointing observations is expected to be six thousand during life of the cryogen. After loss of cryogen (Phase III), only the near-infrared pointing observation will be continued for the remainder of the satellite's life. 

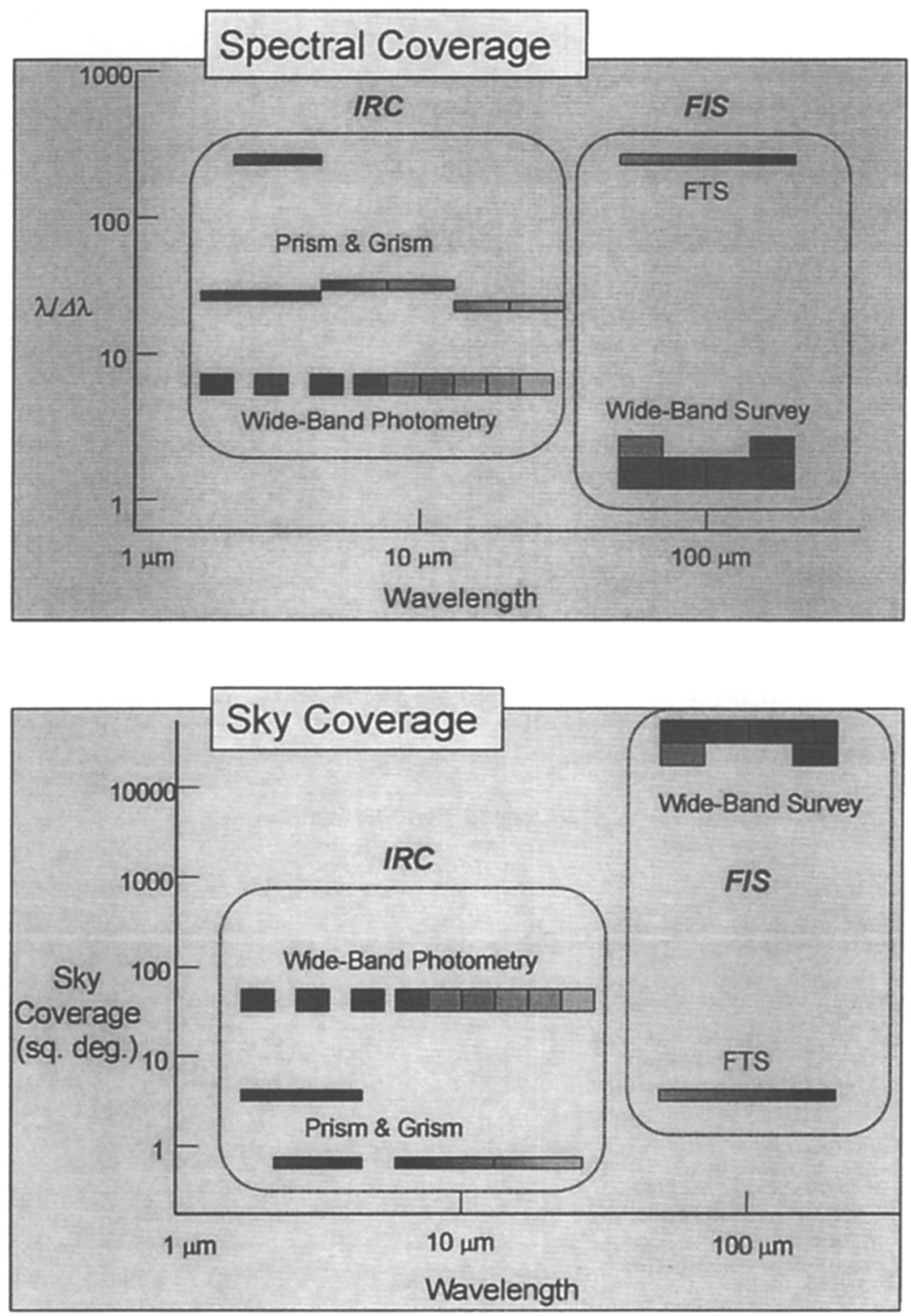

Figure 8. Observational capability of the ASTRO-F mission 

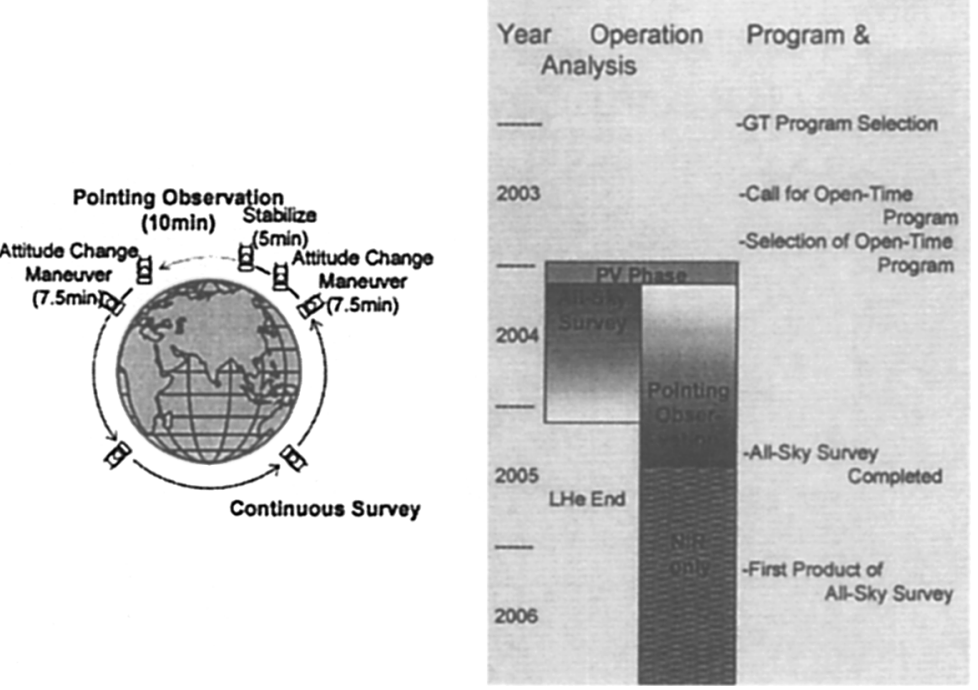

Figure 9. Orbit and time-line of the $A S T R O-F$ mission

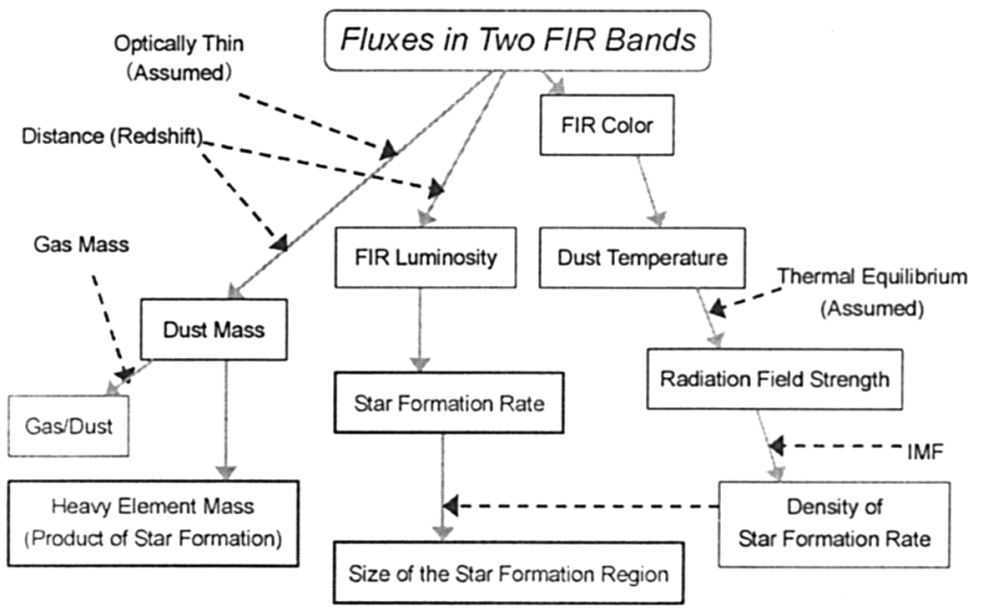

Figure 10. Roadmap to derive scientific information from the observed flux levels in the two far-infrared bands and the use of additional data 


\section{Scientific Objectives}

The primary scientific purpose of the ASTRO-F mission is to investigate the processes leading to the formation and evolution of galaxies in the early universe. In the far-infrared region, the second all-sky survey following that of $I R A S$ will be conducted with remarkably improved performance; more than ten times higher sensitivity for point sources $(20-110 \mathrm{mJy})$, several times better spatial resolution (30-50 arcsec), and an added, longer wavelength band (up to $170 \mu \mathrm{m})$. The final point source catalog is expected to include distant ultraluminous galaxies, starburst galaxies, proto-planetary disks, and other sources. The expected number of detected point sources, mainly external galaxies, is more than ten million, which should include more than ten thousand distant starbursts at $z>1$ (Takeuchi et al. 1999).

In addition to the all-sky survey, the IRC will conduct a deep wide-sky survey of pre-selected parts of the sky at 2 to 25 microns. IRC has six photometric bands and low-resolution grism spectroscopy.

The FIS all-sky survey catalog and the selected field images taken by the IRC and by the FIS spectrometer will not only enable us to investigate the history of galaxy evolution in the early universe with statistical means but also contribute to detailed observations by SIRTF (Fanson et al. 1998), FIRST (Pilbratt 1998), NGST (Seery et al. 1998), HIIL2 (Nakagawa et al. 1998), SOFIA (Becklin 1998), and other future observatory-type missions and ground-based telescopes.

Acknowledgments. The author is deeply indebted to all members of the $A S T R O-F$ (IRIS) project. The figures were originally constructed by $\mathrm{H}$. Murakami, $\mathrm{H}$. Matsuhara, and $\mathrm{M}$. Kawada, and the author thanks them for their courtesy.

\section{References}

Becklin, E. E. 1998, SPIE, 3356, 492

COBE DIRBE Explanatory Supplement. 1994, available at nssdca.gsfc.nasa.gov

Fanson, J. L., et al. 1998, SPIE, 3356, 478

Kawada, M. 1998, SPIE, 3354, 905

Kessler, M. 1996, A\&A, 315, L27

Matsuhara, H. 1998, SPIE, 3354, 915

Murakami, H., et al. 1996, PASJ, 48, L41

Murakami, H. 1998, SPIE, 3356, 471

Nakagawa, T., et al. 1998, SPIE, 3356, 462

Neugebauer, G., et al. 1984, ApJ, 278, L1

Onaka, T., et al. 1998, SPIE, 3354, 900

Pilbratt, G. L. 1998, SPIE, 3356, 452

Seery, B. D., \& Smith, E. P. 1998, SPIE, 3356, 2

Shibai, H. 2000, Adv. Sp. Res., 25, 2273

Takeuchi, T. 1999, PASP, 51, 81 


\section{Discussion}

Tim Hawarden: In the later part of the mission, after the liquid helium runs out, do you expect the near-infrared instruments to retain full performance?

Hiroshi Shibai: The expected focal plane instrument temperature after helium exhaustion is $20-25 \mathrm{~K}$. So, the InSb array will work well. 